TRANSACTIONS OF THE

AMERICAN MATHEMATICAL SOCIETY

Volume 351, Number 4, April 1999, Pages 1691-1704

S 0002-9947(99)02095-4

\title{
HAAR MEASURE AND THE ARTIN CONDUCTOR
}

\author{
BENEDICT H. GROSS AND WEE TECK GAN
}

\begin{abstract}
Let $G$ be a connected reductive group, defined over a local, nonarchimedean field $k$. The group $G(k)$ is locally compact and unimodular. In On the motive of a reductive group, Invent. Math. 130 (1997), by B. H. Gross, a Haar measure $\left|\omega_{G}\right|$ was defined on $G(k)$, using the theory of Bruhat and Tits. In this note, we give another construction of the measure $\left|\omega_{G}\right|$, using the Artin conductor of the motive $M$ of $G$ over $k$. The equivalence of the two constructions is deduced from a result of G. Prasad.
\end{abstract}

\section{The Root Datum and Motive of $G$}

In this section, $k$ is an arbitrary field and $G$ is a connected reductive group over $k$. We let $\bar{k}$ be an algebraic closure of $k, k_{s}$ the separable closure of $k$ in $\bar{k}$, and $\Gamma=\operatorname{Gal}\left(k_{s} / k\right)$.

Let $T \subset B \subset G$ be a maximal torus, contained in a Borel subgroup, defined over $k_{s}$. Let $\Psi=\Psi(G, B, T)$ be the based root datum defined by this choice. We recall (cf. [Sp], pg. 3-12) that:

$$
\Psi=\left(X^{\bullet}(T), \Delta^{\bullet}(T, B), X_{\bullet}(T), \Delta_{\bullet}(T, B)\right),
$$

with $X^{\bullet}(T)$ and $X_{\bullet}(T)$ the character and cocharacter groups of $T$ respectively, and $\Delta^{\bullet}$ and $\Delta_{\text {. }}$ the simple roots and coroots determined by $B$ respectively. Let $W=N_{G}(T) / T$ be the Weyl group of $\Psi$. The finite group $W$ acts as automorphisms of $X^{\bullet}(T)$, and is generated by the reflections:

$$
s_{\alpha}(x)=x-\left\langle x, \alpha^{\vee}\right\rangle \alpha
$$

for $\alpha \in \Delta^{\bullet}$

The Galois group $\Gamma$ acts as automorphisms of $\Psi$, i.e. as automorphisms of the group $X^{\bullet}(T)$ preserving the finite set $\Delta^{\bullet}$, as follows. If $\sigma \in \Gamma$, then we can find $g \in G\left(k_{s}\right)$ such that

$$
\begin{aligned}
& \operatorname{Int}(g)(\sigma T)=g \sigma(T) g^{-1}=T, \\
& \operatorname{Int}(g)(\sigma B)=g \sigma(B) g^{-1}=B,
\end{aligned}
$$

with $g$ well-defined up to left multiplication by $T\left(k_{s}\right)$. Hence it induces a welldefined automorphism

$$
\psi(\sigma): X^{\bullet}(T) \longrightarrow X^{\bullet}(T)
$$

preserving $\Delta^{\bullet}$. Hence we get a group homomorphism $\psi: \Gamma \longrightarrow \operatorname{Aut}(\Psi)$. Via $\psi, \Gamma$ acts on $A u t(\Psi)$ by inner automorphisms.

Received by the editors March 4, 1997.

1991 Mathematics Subject Classification. Primary 11E64.

(C)1999 American Mathematical Society 
Similarly, if $f: G \longrightarrow G$ is any automorphism of $G$ over $k_{s}$, it induces an automorphism $\psi(f)$ of $\Psi$, which depends only on the image of $f$ in the quotient group $\operatorname{Out}_{k_{s}}(G)$ of outer automorphisms. The resulting map $\operatorname{Out}_{k_{s}}(G) \longrightarrow A u t(\Psi)$ is an isomorphism which respects the respective Galois actions on the two groups (cf. [Sp], pg. 10).

The Galois group $\Gamma$ also acts on $W$, via the formula

$$
\sigma\left(s_{\alpha}\right)=s_{\sigma(\alpha)}
$$

and the semi-direct product $W \rtimes \Gamma$ acts on the rational vector space

$$
E=X^{\bullet}(T) \otimes \mathbb{Q} \text {. }
$$

Let $R=\operatorname{Sym}_{\bullet}(E)^{W}$, which is a graded $\mathbb{Q}[\Gamma]$-module. Let $R_{+}$be the ideal of elements of positive degree in $R$, and define

$$
V=R_{+} / R_{+}^{2}=\bigoplus_{d \geq 1} V_{d}
$$

This is a graded $\mathbb{Q}[\Gamma]$-module, and Chevalley proved that $\operatorname{dim}(V)=\operatorname{dim}(E)$ (cf. $[\mathrm{Ch}])$. Steinberg extended the proof to show that $E$ and $V$ are isomorphic $\Gamma$ modules (cf. [St], pg. 22). We sketch a proof of this result that does not involve the classification of irreducible root systems.

Proposition 1.6. The $\mathbb{Q}[\Gamma]$-modules $E$ and $V$ are isomorphic.

Proof. By the criterion in [Se, pg. 104], it suffices to show that for all $\sigma \in \Gamma$, the fixed spaces $E^{\sigma}$ and $V^{\sigma}$ have the same dimension.

For any graded $\Gamma$-module $A=\bigoplus A_{m}$, we define the Poincare series of $\sigma$ by

$$
P(A, \sigma)(t)=\sum \operatorname{tr}\left(\sigma \mid A_{m}\right) t^{m}
$$

Then $P(A \otimes B)=P(A) P(B)$. Steinberg showed that there is an isomorphism of graded $\Gamma$-modules:

$$
S^{\bullet}(E) \cong S^{\bullet}\left(\oplus V_{d}\right) \otimes A .
$$

Here $A$ is finite dimensional, with basis $\left\{b_{w}\right\}_{w \in W}$, and $\Gamma$-action given by:

$$
\sigma\left(b_{w}\right)=b_{\sigma(w)} .
$$

The degree of $b_{w}$ is the length $l(w)$ of $w$, with respect to the generators $s_{\alpha}$ furnished by $\Delta^{\bullet}$. This isomorphism yields the following identity of Poincare series:

$$
\operatorname{det}(1-\sigma t \mid E)^{-1}=\prod_{d \geq 1} \operatorname{det}\left(1-\sigma t^{d} \mid V_{d}\right)^{-1} \cdot \sum_{w \in W^{\sigma}} t^{l(w)}
$$

In particular, the quotient

$$
\frac{\prod_{d \geq 1} \operatorname{det}\left(1-\sigma t^{d} \mid V_{d}\right)}{\operatorname{det}(1-\sigma t \mid E)}
$$

is a polynomial $P(t)$, with $P(1) \neq 0$. Hence $\operatorname{dim}\left(V^{\sigma}\right)=\sum_{d \geq 1} \operatorname{dim}\left(V_{d}^{\sigma}\right)=\operatorname{dim}\left(E^{\sigma}\right)$, as required.

As in [Gr], we define the motive $M$ of $G$ as the Artin-Tate motive

$$
M=\bigoplus_{d \geq 1} V_{d}(1-d)
$$


over $k$. This depends only on the isogeny class of the quasi-split inner form $G_{q s}$ of $G$ over $k$. Indeed, if $T_{q s}$ is a maximal torus contained in a Borel subgroup $B_{q s} \subset G_{q s}$ over $k$, then,

$$
E \cong X^{\bullet}\left(T_{q s}\right) \otimes \mathbb{Q}
$$

as a $W \rtimes \Gamma$-module (cf. [Sp], pg. 12).

We also define the invariant

$$
d(G) \in \operatorname{Hom}\left(\Gamma, \mathbb{Z}^{\times}\right)=H^{1}\left(\Gamma, \mathbb{Z}^{\times}\right)
$$

as the character of $\Gamma$ on $\wedge^{t o p} X^{\bullet}(T)$, or equivalently as the representation $\operatorname{det}(E)$ of $\Gamma$. This is analogous to, but simpler than Kottwitz's invariant $e(G) \in H^{2}\left(\Gamma, \mu_{2}\right)$ (cf. $[\mathrm{K}]$ ).

The canonical ring homomorphism $c h: \mathbb{Z} \longrightarrow k$ induces a map $\mathbb{Z}^{\times} \longrightarrow \mu_{2}$. We let

$$
\delta(G) \in H^{1}\left(\Gamma, \mu_{2}\right)=k^{\times} / k^{\times 2}
$$

be the image of the invariant $d(G)$. This is trivial when $\operatorname{char}(k)=2$, and can be computed in general as follows. Let $K$ be the étale $k$-algebra of dimension 2 corresponding to $d(G)$. Write $K=k+k \alpha$, and suppose $\alpha$ satisfies the non-zero quadratic polynomial $a \alpha^{2}+b \alpha+c=0$ over $k$. Then $\delta(G) \equiv b^{2}-4 a c\left(\bmod k^{\times 2}\right)$.

\section{Automorphisms of $G$}

Let $f$ be an automorphism of $G$ over $k_{s}$. Let $\psi(f)$ be the corresponding automorphism of the based root datum $\Psi$, and let Lie $(f)$ be the corresponding automorphism of the Lie algebra $\mathfrak{g}$ over $k_{s}$. The former depends only on the image of $f$ in $\mathrm{Out}_{k_{s}}(G)$; similarly we have the following:

Lemma 2.1. The automorphism $\wedge^{\text {top }} \operatorname{Lie}(f)$ of $\wedge^{\text {top }} \mathfrak{g}$ depends only on the image of $f$ in Out $_{k_{s}}(G)$.

Proof. The action of inner automorphisms on $\wedge^{t o p} \mathfrak{g}$ gives a homomorphism $G^{a d} \longrightarrow$ $\mathbb{G}_{m}$ of algebraic groups over $k$. This is trivial as $G^{a d}$ is connected with trivial center.

Proposition 2.2.

$$
\operatorname{ch}(\operatorname{det}(\psi(f)))=\operatorname{det}(\operatorname{Lie}(f)) \in \operatorname{ch}\left(\mathbb{Z}^{\times}\right)=\mu_{2}(k) .
$$

Proof. Let $\left\{T, B, X_{\alpha}: \alpha \in \Delta^{\bullet}\right\}$ be a pinning of $G$ over $k_{s}$, where $X_{\alpha}$ is a basis of the one-dimensional root space $\mathfrak{g}_{\alpha}$. By the previous lemma, we may assume that the automorphism $f$ preserves the pinning (cf. [Sp], pg. 10). Then Lie $(f)$ preserves a Chevalley basis of $\mathfrak{g}$ over $k_{s}$ (cf. [B-T], pg. 53-54).

Let $\mathfrak{t}$ be the Lie algebra of $T$, and $\mathfrak{n}^{ \pm}$the nilpotent Lie algebra spanned by the positive and negative roots with respect to $B$. Then $\operatorname{Lie}(f)$ preserves the triangular decomposition

Furthermore,

$$
\mathfrak{g}=\mathfrak{t} \oplus \mathfrak{n}^{+} \oplus \mathfrak{n}^{-} .
$$

$$
\operatorname{det}(\operatorname{Lie}(f) \mid \mathfrak{t})=\operatorname{ch}(\operatorname{det}(\psi(f)))
$$

as $\mathfrak{t}=X_{\bullet}(T) \otimes k$. Since the permutation induced by Lie $(f)$ on the positive elements of the Chevalley basis is the same as that on the negative elements, we have

$$
\operatorname{det}\left(\operatorname{Lie}(f) \mid \mathfrak{n}^{+}\right) \cdot \operatorname{det}\left(\operatorname{Lie}(f) \mid \mathfrak{n}^{-}\right)=1 .
$$

This completes the proof. 
Recall that the invariant differential forms of top degree on $G$ over an extension $L$ of $k$ form a one-dimensional $L$-vector space, which is the dual of $\wedge^{t o p} \mathfrak{g}_{L}$. We will refer to an element of this space as an invariant differential on $G$.

Corollary 2.3. If $\omega$ is an invariant differential on $G$ over $k_{s}$, and $f$ is any automorphism of $G$ over $k_{s}$, then $f^{*}(\omega)=\operatorname{ch}(\operatorname{det}(\psi(f))) \omega$.

\section{The Split Group}

Let $G_{0}$ be a split group over $k$, whose root datum is isomorphic to $\Psi$. Such a group exists by $[\mathrm{B}-\mathrm{T}]$, and we may choose an isomorphism

$$
\varphi: G \longrightarrow G_{0}
$$

defined over $k_{s}$.

For each $\sigma \in \Gamma$, the element

$$
f(\sigma)=\varphi^{-1} \circ \sigma(\varphi)
$$

defines an automorphism of $G$ over $k_{s}$. The map $f: \Gamma \longrightarrow A_{k_{k_{s}}}(G)$ is a 1cocycle, whose class in $H^{1}\left(\Gamma, A_{u} t_{k_{s}}(G)\right)$ is independent of the choice of $\varphi$. The map $\sigma \mapsto \psi(f(\sigma))$ is then a 1-cocycle with values in $A u t(\Psi)$. Composing this with

$$
\text { det }: \operatorname{Aut}(\Psi) \longrightarrow \mathbb{Z}^{\times}
$$

we get a group homomorphism

Lemma 3.5.

$$
\begin{gathered}
\Gamma \longrightarrow \mathbb{Z}^{\times}, \\
\sigma \mapsto \operatorname{det}(\psi(f(\sigma))) .
\end{gathered}
$$

$$
\operatorname{det}(\psi(f(\sigma)))=d(G)(\sigma) \in \mathbb{Z}^{\times} .
$$

Proof. By (1.8) and Lemma 2.1, it suffices to prove this for $G$ quasi-split over $k$. Hence we can assume that $T$ and $B$ are defined over $k$. Let $T_{0} \subset B_{0}$ be a maximal torus of $G_{0}$ contained in a Borel subgroup, with $T_{0}$ and $B_{0}$ defined over $k$. Twisting by an inner automorphism of $G_{0}$ if necessary, we can suppose that the isomorphism $\varphi$ in (3.1) maps $T$ and $B$ to $T_{0}$ and $B_{0}$ respectively. Then using $\varphi$, we can identify $G\left(k_{s}\right), T\left(k_{s}\right)$ and $B\left(k_{s}\right)$ with $G_{0}\left(k_{s}\right), T_{0}\left(k_{s}\right)$ and $B_{0}\left(k_{s}\right)$ respectively. Now suppose that $G(k)$ is the fixed-point set of the $\Gamma$-action $g \mapsto \sigma(g)$ on $G\left(k_{s}\right)=G_{0}\left(k_{s}\right)$. Then $G_{0}\left(k_{s}\right)$ is the fixed-point set of the $\Gamma$-action $g \mapsto f(\sigma)(\sigma(g))=\rho(\sigma)(g)$. Now the action of $\psi(\rho(\sigma))$ on $X^{\bullet}(T)=X^{\bullet}\left(T_{0}\right)$ is trivial, since $G_{0}$ is split. Hence, for any $\chi \in X^{\bullet}(T)$, we have

$$
\begin{aligned}
\psi(f(\sigma)) \chi & =\psi(\sigma)^{-1} \psi(\sigma) \psi(f(\sigma)) \chi \\
& =\psi(\sigma)^{-1} \psi(\rho(\sigma)) \chi \\
& =\psi(\sigma)^{-1} \chi
\end{aligned}
$$

Hence the action of $\psi(f(\sigma))$ on $X^{\bullet}(T)$ is the same as that of $\psi(\sigma)^{-1}$. This implies the result.

Proposition 3.6. Let $\omega_{0}$ be an invariant differential on $G_{0}$ over $k$, and let $\omega=$ $\varphi^{*}\left(\omega_{0}\right)$ on $G$ over $k_{s}$. Then for all $\sigma \in \Gamma$,

$$
\sigma(\omega)=\delta(G)(\sigma) \cdot \omega
$$

where $\delta(G)$ is the character of $\Gamma$ with values in $\mu_{2}(k)$ defined by $(1.10)$. 
Proof. We have $\sigma(\omega)=\operatorname{ch}(\operatorname{det}(\psi(f(\sigma)))) \omega$ by Corollary 2.3. By the previous lemma,

$$
\operatorname{det}(\psi(f(\sigma)))=d(G)(\sigma) \in \mathbb{Z}^{\times}
$$

So we have

$$
\operatorname{ch}\left(\operatorname{det}(\psi(f(\sigma)))=\delta(G)(\sigma) \in k^{\times} / k^{\times 2} .\right.
$$

Corollary 3.7. Let $D \in k^{\times} / k^{\times 2}$ represent the class of $\delta(G)$. Then $\omega / \sqrt{D}$ is an invariant differential on $G$ over $k$.

Proof. Indeed, $\sigma(\sqrt{D})=\delta(G)(\sigma) \sqrt{D}$, so the differential $\omega / \sqrt{D}$ is fixed by $\Gamma$. Note that when $\operatorname{char}(k)=2, D$ is in $k^{\times 2}$ and so $\sqrt{D} \in k^{\times}$.

\section{The Artin Conductor of $M$}

We now assume that $k$ is a local, non-archimedean field, with ring of integers $A$ and uniformizer $\pi$. We let $q=\#(A / \pi A)$, and normalize the valuation on $k^{\times}$so that $v(\pi)=1$, and the absolute value so that $|\alpha|=q^{-v(\alpha)}$. We adopt the convention that $|0|=0$.

Let $V$ be a continuous finite dimensional complex representation of $\Gamma$. We define the Artin conductor $a(V) \geq 0$ in $\mathbb{Z}$ as follows. Let $L$ be the fixed field of the kernel of the map $\Gamma \longrightarrow G L(V)$; let $\Delta=\operatorname{Gal}(L / k)$, which is a finite group, and let

$$
\Delta \supset \Delta_{0} \supset \Delta_{1} \supset \ldots
$$

be the decreasing ramification filtration of $\Delta$. Then $\Delta_{0}=I$ is the inertia subgroup and $\Delta_{1}$ the wild inertia subgroup. Let $g_{i}=\# \Delta_{i}$. Then [Se3, pg. 99-101],

$$
a(V)=\sum_{i \geq 0} \frac{g_{i}}{g_{0}} \operatorname{dim}\left(V / V^{\Delta_{i}}\right) .
$$

We have $a(V)=\operatorname{dim}\left(V / V^{I}\right)+b(V)$, where $b(V)$ is a measure of the wild ramification of $V$.

If $V$ is a quadratic character $\chi: \Gamma \longrightarrow \mathbb{Z}^{\times}$, we can refine the integer $a(V)$ slightly, as follows. Let $K$ be the étale $k$-algebra of dimension 2 corresponding to $\chi$, and let $A_{K} \subset K$ be the subring of elements integral over $A$. Then $A_{K}$ is a free $A$-module of rank 2. Writing $A_{K}=A+A \alpha$, we may define $D=D(\alpha)=\operatorname{Tr}(\alpha)^{2}-4 \mathbb{N}(\alpha)$ in $A$. Then $D$ is non-zero, and $[\mathrm{M}-\mathrm{H}]$

$$
a(V)=a(\chi)=v(D) .
$$

If $A_{K}=A+A \alpha^{\prime}$, then $D^{\prime} \equiv D\left(\bmod A^{\times 2}\right)$. Hence we get a class $D_{V}$ in $A / A^{\times 2}$ of valuation $a(V)$; this is the desired refinement.

We define the conductor of the motive $M=\bigoplus_{d \geq 1} V_{d}(1-d)$ of $G$ by the formula:

$$
a(M)=\sum_{d \geq 1}(2 d-1) a\left(V_{d}\right) .
$$

Then $a(M) \geq 0$, with equality if $M=M^{I}$ is unramified.

Proposition 4.4. The conductor $a(M)$ of $M$ and the conductor a $(\operatorname{det} E)$ of the quadratic character $\operatorname{det}(E)=d(G): \Gamma \longrightarrow \mathbb{Z}^{\times}$satisfy

$$
a(M) \equiv a(\operatorname{det} E) \quad(\bmod 2) .
$$


Proof. Clearly,

$$
a(M) \equiv \sum_{d \geq 1} a\left(V_{d}\right)=a(V) \quad(\bmod 2) .
$$

By Proposition 1.6, $V \cong E$ as $\mathbb{Q}[\Gamma]$-modules, so $a(V)=a(E)$. Finally, since $E$ is defined over $\mathbb{R}$, a result of Serre [Se2, pg. 698] gives the congruence

$$
a(E) \equiv a(\operatorname{det} E) \quad(\bmod 2)
$$

This result allows us to refine the conductor $a(M)$ as in (4.2). Since $\operatorname{det} E$ is a quadratic character, there is a class $D$ in $A / A^{\times 2}$ with

$$
v(D)=a(\operatorname{det} E)
$$

Moreover, we have

$$
\sigma(\sqrt{D})=\delta(G)(\sigma) \cdot \sqrt{D}
$$

for all $\sigma \in \Gamma$, where $\delta(G): \Gamma \longrightarrow \mu_{2}(k)$. We define the refinement:

$$
D_{M}=D \pi^{a(M)-a(\operatorname{det} E)} \in A / A^{\times 2} .
$$

Corollary 4.6. The class $D_{M}$ in $A / A^{\times 2}$ satisfies

$$
\begin{aligned}
& v\left(D_{M}\right)=a(M), \text { the Artin conductor of } M, \text { and } \\
& \sigma\left(\sqrt{D_{M}}\right)=\delta(G)(\sigma) \cdot \sqrt{D_{M}}
\end{aligned}
$$

for all $\sigma \in \Gamma$.

\section{The HaAr Measure $\left|\omega_{G}\right|$}

We continue to assume that $k$ is local and non-archimedean. Let $G_{0}$ be the split form of $G$ over $k$, and let $\underline{G}_{0}$ be a Chevalley model for $G_{0}$ over $A$. Let $\omega_{0}$ be an invariant differential on $\underline{G}_{0}$ over $A$ with non-zero reduction $(\bmod \pi)$. Then $\omega_{0}$ is determined up to multiplication by an element of $A^{\times}$.

Let $\varphi: G \longrightarrow G_{0}$ be an isomorphism over $k_{s}$, and define

$$
\omega=\varphi^{*}\left(\omega_{0}\right)
$$

on $G$ over $k_{s}$. By the above remarks, and Corollary $2.3, \omega$ is determined up to multiplication by $A^{\times}$, independent of the choice of $\varphi$.

Let $D_{M}$ in $A / A^{\times 2}$ be defined by (4.5). By Proposition 3.6, and Corollaries 3.7 and 4.6 , the invariant differential

$$
\omega_{G}=\omega / \sqrt{D_{M}}=\varphi^{*}\left(\omega_{0}\right) / \sqrt{D_{M}}
$$

on $G$ is defined over $k$, and is well-determined up to multiplication by an element of $A^{\times}$.

Since $|\alpha|=1$ for all $\alpha \in A^{\times}$, the Haar measure

$$
\left|\omega_{G}\right| \text { on } G(k)
$$

is well-defined, independent of the choices of $\omega_{0}$ and $\varphi$. This completes the definition of $\left|\omega_{G}\right|$. 


\section{Properties of $\left|\omega_{G}\right|$}

We have the following properties of the Haar measure $\left|\omega_{G}\right|$ on $G(k)$, when we vary the group $G$ or the local field $k$.

Proposition 6.1. 1) If $G=G_{1} \times G_{2}$, then $\left|\omega_{G}\right|=\left|\omega_{G_{1}}\right| \otimes\left|\omega_{G_{2}}\right|$ on $G(k)=$ $G_{1}(k) \times G_{2}(k)$.

2) If $\varphi: G \longrightarrow G^{\prime}$ is an inner twisting, defined over $k_{s}$, then $\varphi^{*}\left|\omega_{G^{\prime}}\right|=\left|\omega_{G}\right|$ on $G(k)$.

3) If $f: G \longrightarrow G^{\prime}$ is a central isogeny, defined over $k$, and $N_{f}$ is the rank of the finite flat group scheme kerf, then

$$
f^{*}\left|\omega_{G^{\prime}}\right|=\left|N_{f}\right| \cdot\left|\omega_{G}\right| \text { on } G(k) \text {. }
$$

4) If $K$ is a finite separable extension of $k, G_{K}$ is a connected reductive group over $K$, and $G=\operatorname{Res}_{K} / k\left(G_{K}\right)$ is the restriction of scalars to $k$, then $\left|\omega_{G_{K}}\right|{ }_{K}=\left|\omega_{G}\right|$ on $G_{K}(K)=G(k)$.

Remarks. In part (2), the pull-back $\varphi^{*}$ on Haar measures is defined in [L, pg. 69]. In part (3), the groups $G(k)$ and $G\left(k^{\prime}\right)$ are locally isomorphic provided $N_{f}$ is invertible in $k$. If $N_{f}=0$ in $k$, we define $f^{*}\left|\omega_{G^{\prime}}\right|$ to be zero, so that (3) holds trivially.

Proof. Parts (1) and (2) are simple consequences of the definitions, as $M_{G}=M_{G_{1}} \oplus$ $M_{G_{2}}$ in (1) and $M_{G}=M_{G^{\prime}}$ in (2).

For part (3), the equality of motives allows one to reduce to the case when $G$ and $G^{\prime}$ are split over $k$. Let $T \subset B \subset G$ be chosen over $k$, and let $T^{\prime}=f(T) \subset$ $B^{\prime}=f(B)$ in $G^{\prime}$. The central isogeny $f$ then induces an injection:

$$
X_{\bullet}(T) \longrightarrow X_{\bullet}\left(T^{\prime}\right)
$$

which maps $\Delta_{\bullet}$ to $\Delta_{\bullet}^{\prime}$ and has cokernel of order $N_{f}$.

By [Sp, pg. 7], we can define the groups $G$ and $G^{\prime}$, as well as the central isogeny $f$ over $\mathbb{Z}: f_{\mathbb{Z}}: G_{\mathbb{Z}} \longrightarrow G_{\mathbb{Z}}^{\prime}$ from the isogeny of the root data. Then $\operatorname{Lie}\left(f_{\mathbb{Z}}\right)$ is an isomorphism on the non-zero root spaces, and induces an injection $\operatorname{Lie}\left(T_{\mathbb{Z}}\right) \longrightarrow \operatorname{Lie}\left(T_{\mathbb{Z}}^{\prime}\right)$ with kernel of order $N_{f}$. If $\omega_{G}$ and $\omega_{G^{\prime}}$ are bases for the invariant differential over $\mathbb{Z}$, we then have

$$
f_{\mathbb{Z}}^{*}\left(\omega_{G^{\prime}}\right)= \pm N_{f} \cdot \omega_{G} .
$$

The result then follows by specializing to $k$.

For part (4), we have

$$
M_{G}=\operatorname{Ind} d_{\Gamma_{K}}^{\Gamma}\left(M_{G_{K}}\right)
$$

where $\Gamma_{K}$ is the subgroup of $\Gamma$ fixing $K$. Let $\varepsilon_{K / k}$ be the sign character of the permutation representation of $\Gamma$ on $\Gamma / \Gamma_{K}=\operatorname{Hom}\left(K, k_{s}\right)$; let $D_{K / k} \in A / A^{\times 2}$ be associated to the quadratic character $\varepsilon_{K / k}$, and let $f_{K / k}$ be the degree of the residue class extension in $K / k$.

If $\omega_{K}$ is an invariant differential on $G_{K}$ over $K$, then the exterior product

$$
\omega=\frac{\bigwedge_{\sigma \in \Gamma / \Gamma_{K}} \omega_{K}^{\sigma}}{\left(\sqrt{D_{K / k}}\right)^{\operatorname{dim}\left(G_{K}\right)}}
$$

is an invariant differential on $G$ defined over $k$. Note that $G\left(k_{s}\right)=\prod_{\sigma \in \Gamma / \Gamma_{K}} G_{K}^{\sigma}\left(k_{s}\right)$. Now, suppose $\left\{X_{1}, \ldots, X_{n}\right\}$ is a basis of $\mathfrak{g}_{K}$, the Lie algebra of $G_{K}$, such that $\omega_{K}\left(X_{1} \wedge \ldots \wedge X_{n}\right)=1$. Let $\left\{\theta_{1}, \ldots, \theta_{r}\right\}$ be a basis of the free $A$-module $A_{K}$, the ring 
of integers of $K$. Then $\left\{\theta_{i} X_{j}: 1 \leq i \leq r, 1 \leq j \leq n\right\}$ is a basis of $\mathfrak{g}$, the Lie algebra of $G$, and by a direct computation, one sees that:

$$
\bigwedge_{\sigma} \omega_{K}^{\sigma}\left(\bigwedge_{i, j} \theta_{i} X_{j}\right)=D_{K / k}^{\frac{\operatorname{dim}\left(G_{K}\right)}{2}}
$$

Hence, $\left|\omega_{K}\right|_{K}=|\omega|$ as Haar measures on $G_{K}(K)=G(k)$. This is compatible with scaling $\omega_{K}$ by $\beta \in K^{\times}$, as $|\beta|_{K}=\left|\mathbb{N}_{K / k}(\beta)\right|$.

Now write $M_{K}=\bigoplus U_{d}(1-d)$ and $M=\bigoplus V_{d}(1-d)$, with $V_{d}=\operatorname{Ind}\left(U_{d}\right)$. By [Se3, pg. 101],

$$
a\left(V_{d}\right)=f_{K / k} \cdot a\left(U_{d}\right)+\operatorname{dim} U_{d} \cdot a\left(\varepsilon_{K / k}\right) .
$$

Since $\sum(2 d-1) \operatorname{dim} U_{d}=\operatorname{dim}\left(G_{K}\right)$, we have

$$
a(M)=f_{K / k} \cdot a\left(M_{K}\right)+\operatorname{dim}\left(G_{K}\right) \cdot a\left(\varepsilon_{K / k}\right) .
$$

The corresponding result for the refinements $D_{M_{K}}$ of $a\left(M_{K}\right)$ in $A_{K} / A_{K}^{\times 2}$ and $D_{M}$ of $a(M)$ in $A / A^{\times 2}$ is then

$$
D_{M} \equiv \mathbb{N}_{K / k}\left(D_{M_{K}}\right) \cdot D_{K / k}^{\operatorname{dim}\left(G_{K}\right)} .
$$

Now if $G_{0, K}$ is a split form of $G_{K}, \varphi_{K}: G_{K} \longrightarrow G_{0, K}$ an isomorphism over $k_{s}$, and $\omega_{0, K}$ an invariant differential on $G_{0, K}$ with good reduction, then by definition

$$
\omega_{G_{K}}=\frac{\varphi_{K}^{*}\left(\omega_{0, K}\right)}{\sqrt{D_{M_{K}}}}
$$

As observed above, the form on $G$ over $k$ which gives the same Haar measure on $G(k)=G_{K}(K)$ as $\omega_{G_{K}}$ is given by

$$
\omega=\frac{\bigwedge \varphi_{K}^{*}\left(\omega_{0, K}\right)^{\sigma}}{\sqrt{\mathbb{N}_{K / k}\left(D_{M_{K}}\right) \cdot D_{K / k}^{\operatorname{dim}\left(G_{K}\right)}}}=\frac{\varphi^{*}\left(\omega_{0}\right)}{\sqrt{D_{M}}}=\omega_{G} .
$$

This completes the proof.

\section{Comparison with Bruhat-Tits Theory}

First, we assume that $G$ is quasi-split over $k$. In [Gr, §4], a Haar measure $\left|\omega_{G}^{\prime}\right|$ was defined on $G(k)$. The definition used the theory of special points in the building of $G$, and models over $A$. If $G$ is split, then $\left|\omega_{G}^{\prime}\right|=\left|\omega_{G}\right|$ by definition. It seems likely that this is true in general. The key case, when $G$ is absolutely quasi-simple and simply connected, was treated by Prasad $[\mathrm{P}]$. We deduce what we can from his results here.

Since the Haar measure $\left|\omega_{G}^{\prime}\right|$ is also defined using an invariant differential $\omega_{G}^{\prime}$ on $G$ over $k$, we have

$$
\left|\omega_{G}^{\prime}\right|=\lambda_{G}\left|\omega_{G}\right|
$$

with $\lambda_{G}$ in the subgroup $q^{\mathbb{Z}}$ of $\mathbb{R}_{+}^{\times}$.

Proposition 7.2. We have $\lambda_{G}=1$ if $G$ is unramified over $k$. Furthermore,

1) $\lambda_{G_{1} \times G_{2}}=\lambda_{G_{1}} \lambda_{G_{2}}$.

2) $\lambda_{G}=\lambda_{G^{\prime}}$ if $G$ and $G^{\prime}$ are separably isogeneous over $k$.

3) $\lambda_{G_{K}}=\lambda_{G}$ if $G=\operatorname{Res}_{K / k}\left(G_{K}\right)$. 
Proof. If $G$ is unramified, $a(M)=0$, and $D_{M}$ is in $A^{\times} / A^{\times 2}$. Also, $\omega_{G}^{\prime}$ is defined using a hyperspecial point in the building of $G$, which is a special vertex in the building over the maximal unramified extension in $k_{s}$. Hence $\omega_{G}=\varphi^{*}\left(\omega_{0}\right) / \sqrt{D_{M}}$ is a unit multiple of $\omega_{G}^{\prime}$, and $\lambda_{G}=1$.

Properties (1) - (4) of Proposition 6.1 hold for $\left|\omega_{G}^{\prime}\right|$, which implies properties (1) - (3) in the proposition.

Corollary 7.3. If char $(k)=0$, then $\left|\omega_{G}\right|=\left|\omega_{G}^{\prime}\right|$.

If char $(k)=p$, then $\left|\omega_{G}\right|=\left|\omega_{G}^{\prime}\right|$ if $G$ is a torus with Galois splitting field of degree prime to $p$, or if $G$ is semi-simple with fundamental group of order prime to $p$.

Proof. If the characteristic of $k$ is zero, any central isogeny is separable. By Proposition 7.2 , it suffices to prove the equality $\left|\omega_{G}\right|=\left|\omega_{G}^{\prime}\right|$ for $G$ semi-simple, simplyconnected, and for $G$ a torus. Indeed, $G$ is isogeneous to the product of the simplyconnected cover of its derived group, and its connected center.

If $G$ is semi-simple and simply-connected, then $G$ is isomorphic to a product $\prod \operatorname{Res}_{K_{i} / k}\left(G_{i}\right)$, with each $G_{i}$ absolutely quasi-simple over $K_{i}$. Again by Proposition 7.2, it suffices to prove the equality for $G$ absolutely quasi-simple. This is the content of Theorem 1.6 of Prasad [P].

If $G$ is a torus, there is an integer $n$ such that $G^{n} \times \prod \operatorname{Res}_{K_{i} / k} \mathbb{G}_{m}$ is isogeneous to $\prod \operatorname{Res}_{K_{j} / k} \mathbb{G}_{m}$ by a Theorem of Ono [O, Thm 1.5.1, pg. 114]. Since the result is true for $\mathbb{G}_{m}$, it is true for $G^{n}$. So $\lambda_{G}^{n}=1$; since $\lambda$ is positive, we also have: $\lambda_{G}=1$.

If the characteristic of $k$ is $p$, and $G$ is a torus with Galois splitting field of degree prime to $p$, then the same Theorem of Ono alluded to above says that the isogeny from $G^{n} \times \prod \operatorname{Res}_{K_{i} / k} \mathbb{G}_{m}$ to $\prod \operatorname{Res}_{K_{j} / k} \mathbb{G}_{m}$ can be chosen to be separable. Hence the same argument as above works to give the result.

If $G$ is semi-simple with fundamental group of order prime to $p$, the isogeny $\tilde{G} \longrightarrow G$ from the simply-connected cover is separable. So it suffices to check the result for $\tilde{G}$. By the above argument, we may assume that $G=\tilde{G}$ is absolutely quasi-simple, where the result follows from Prasad $[\mathrm{P}]$.

Now if $G$ is not necessarily quasi-split, choose an inner twisting $\varphi: G \longrightarrow G_{q s}$, where $G_{q s}$ is the quasi-split inner form of $G$. In [Gr], the measure $\left|\omega_{G}^{\prime}\right|$ on $G(k)$ was defined to be $\varphi^{*}\left|\omega_{G_{q s}}^{\prime}\right|$. Then we have

Corollary 7.4. If $\operatorname{char}(k)=0$, then $\left|\omega_{G}\right|=\left|\omega_{G}^{\prime}\right|$. Furthermore, let $J \subset G(k)$ be an Iwahori subgroup. Then,

$$
\int_{J}\left|\omega_{G}\right|=q^{-N} \cdot \operatorname{det}\left(1-F w_{G} \mid E(1)^{I}\right)
$$

with $N=\sum(d-1) d i m V_{d}^{I}, F$ the geometric Frobenius in $\Gamma / I$ with eigenvalue $q^{-1}$ on $\mathbb{Q}(1)$, and $w_{G}$ the element of the Weyl group $W^{I}$ associated to the inner twisting $\varphi: G \longrightarrow G_{q s}$ over the maximal unramified extension of $k$.

Proof. This was established for $\left|\omega_{G}^{\prime}\right|$ in [Gr, §4]. Note that if $G=G_{q s}$ is quasi-split, then $w_{G}=1$.

\section{The Space of Haar Measures}

Let $P_{G}$ be the one-dimensional real vector space of invariant measures on $G(k)$, and let $P_{G}^{+}$be the cone of positive Haar measures in $P_{G}$. We define, from $\left|\omega_{G}\right|$, the 
following element of $P_{G}^{+}$:

$$
\mu_{G}=\left|\omega_{G}\right| \cdot q^{-a(M) / 2} .
$$

Let $\varphi: G \longrightarrow G^{\prime}$ be an isomorphism over $k_{s}$. We define an $\mathbb{R}$-linear map

$$
\varphi^{*}: P_{G^{\prime}} \longrightarrow P_{G}
$$

as follows. Let $\mu^{\prime}$ be an element of $P_{G^{\prime}}$, and write $\mu^{\prime}=c\left|\omega^{\prime}\right|$, for some invariant differential $\omega^{\prime}$ on $G^{\prime}$ over $k$, and $c \in \mathbb{R}$. Let $d \in k^{\times} / k^{\times 2}$ be the class of the map:

$$
\delta(G) \cdot \delta\left(G^{\prime}\right): \Gamma \longrightarrow \mu_{2}(k) .
$$

It follows from Proposition 3.6 that the differential

$$
\omega=\varphi^{*}\left(\omega^{\prime}\right) / \sqrt{d}
$$

on $G$ is defined over $k$. We then define

$$
\varphi^{*}\left(\mu^{\prime}\right)=c|\omega| \cdot|d|^{\frac{1}{2}} \in P_{G} .
$$

This is independent of the choice of $\omega^{\prime}$ and $d$, and we have the following result.

Proposition 8.4. The map $\varphi^{*}: P_{G^{\prime}} \longrightarrow P_{G}$ is an $\mathbb{R}$-linear isomorphism, which maps $P_{G^{\prime}}^{+}$to $P_{G}^{+}$. Furthermore, $\varphi^{*}\left(\mu_{G^{\prime}}\right)=\mu_{G}$.

The isomorphism $P_{G^{\prime}} \cong P_{G}$ is independent of the choice of the isomorphism $\varphi: G \longrightarrow G^{\prime}$ over $k_{s}$.

Proof. All the statements will follow once we show that $\varphi^{*}\left(\mu_{G^{\prime}}\right)=\mu_{G}$. This identity follows from a comparison of $G$ and $G^{\prime}$ with the split group $G_{0}$ over $k_{s}$. Indeed, $\mu_{G_{0}}=\left|\omega_{0}\right|$, and for $\varphi: G \longrightarrow G_{0}$, we have

$$
\begin{aligned}
\mu_{G} & =\left|\omega_{G}\right| q^{-a(M) / 2} \\
& =\left|\varphi^{*}\left(\omega_{0}\right) / \sqrt{D_{M_{G}}}\right| \cdot\left|D_{M_{G}}\right|^{\frac{1}{2}} \\
& =\varphi^{*}\left(\mu_{G_{0}}\right) .
\end{aligned}
$$

\section{Global Measure}

In this section, we assume that $k$ is a global field. Let $\omega$ be a non-zero invariant differential on $G$ over $k$, and let $|\omega|_{v}$ be the associated Haar measure on $G\left(k_{v}\right)$, for each place $v$. We define the global conductor $f(M)$ of the motive $M$ of $G$ by the formula:

$$
f(M)=\prod_{v \text { finite }} q_{v}^{a\left(M / k_{v}\right)} .
$$

This product is finite because for almost all $v, G$ is unramified over $k_{v}$ and $a\left(M / k_{v}\right)=0$. The conductor $f(M)$ is an integer $\geq 1$. If $G$ is an inner form of a split group over $k$, then $f(M)=1$.

If $v$ is finite, let $\left|\omega_{G_{v}}\right|$ be the Haar measure on $G\left(k_{v}\right)$ defined in $\S 5$. If $v$ is archimedean, let $\left|\omega_{G_{v}}\right|$ be the measure on $G\left(k_{v}\right)$ defined in [Gr, §11]. In the archimedean case, we can also define $\left|\omega_{G_{v}}\right|$ as follows. Let $G_{0}$ be the split form of $G$ over $k_{v}$, and $G_{0, \mathbb{Z}}$ the Chevalley model for $G_{0}$ over $\mathbb{Z}$. Let $\omega_{0}$ be an invariant differential on $G_{0}$ which generates the free $\mathbb{Z}$-module $\operatorname{Hom}\left(\wedge^{\text {top }} \operatorname{Lie}\left(G_{0, \mathbb{Z}}\right), \mathbb{Z}\right)$. Then 
$\omega_{0}$ is determined up to sign. If $\varphi: G \longrightarrow G_{0}$ is an isomorphism over $k_{s}$, and $K$ (respectively $K_{0}$ ) is the maximal compact subgroup of $G$ (respectively $G_{0}$ ), then,

$$
\omega_{G_{v}}=\frac{\varphi^{*}\left(\omega_{0}\right)}{i^{\operatorname{dim}(G / K)-\operatorname{dim}\left(G_{0} / K_{0}\right)}}
$$

is defined on $G$ over $k_{v}$, and is determined up to sign. The Haar measure $\left|\omega_{G_{v}}\right|$ is thus well-defined.

Proposition 9.3. We have $|\omega|_{v}=\left|\omega_{G_{v}}\right|$ for almost all $v$, and the following product formula holds:

$$
\prod_{v} \frac{\left|\omega_{G_{v}}\right|}{|\omega|_{v}}=f(M)^{\frac{1}{2}} \text { in } \mathbb{R}_{+}^{\times} .
$$

Proof. For almost all $v, G$ is unramified over $k_{v}$, and $\omega$ generates the $A_{v}$-module of invariant differentials on the reductive model $\underline{G}$ over $A_{v}$. At these places, $|\omega|_{v}=$ $\left|\omega_{G_{v}}\right|$.

For $v$ finite, let $\mu_{G_{v}}=\left|\omega_{G_{v}}\right| q^{-a\left(M / k_{v}\right) / 2}$ as in (8.1). For $v$ archimedean, let $\mu_{G_{v}}=\left|\omega_{G_{v}}\right|$. Then the product formula is equivalent to the statement

$$
\prod_{v} \frac{\mu_{G_{v}}}{|\omega|_{v}}=1
$$

This is independent of the choice of $\omega \neq 0$, by the usual product formula: $\prod_{v}|\alpha|_{v}=$ 1 , for $\alpha \in k^{\times}$.

We first prove (9.4) for $G=G_{0}$ split over $k$. In this case, we take $\omega_{0}$ to generate the Chevalley differentials over $\mathbb{Z}$; then $\omega_{0}$ is determined up to sign, and $\left|\omega_{0}\right|_{v}=$ $\left|\omega_{G_{v}}\right|=\mu_{G_{v}}$ for all $v$. Hence (9.4) holds, because all the terms are 1 .

Now let $G$ be arbitrary, and choose an isomorphism $\varphi: G \longrightarrow G_{0}$ with the split form over $k_{s}$. Let $d \in k^{\times} / k^{\times 2}$ be in the class of $\delta(G)$, let $\omega_{0}$ be as above, and let $\omega=\varphi^{*}\left(\omega_{0}\right) / \sqrt{d}$ over $k$. Then we have, for all $v$

$$
\frac{\mu_{G_{v}}}{|\omega|_{v}}=|d|_{v}^{\frac{1}{2}} \cdot \frac{\mu_{\left(G_{0}\right)_{v}}}{\left|\omega_{0}\right|_{v}}=|d|_{v}^{\frac{1}{2}} .
$$

Since $\prod_{v}|d|_{v}^{\frac{1}{2}}=1$, the proposition is proved.

Remarks. This gives a proof of Theorem 11.5 in [Gr, §11], when $k$ is a number field. Indeed, we have shown in $\S 7$ that $\left|\omega_{G}\right|=\left|\omega_{G}^{\prime}\right|$, where $\left|\omega_{G}^{\prime}\right|$ is the Haar measure defined in $[\mathrm{Gr}]$. Also the constant $\varepsilon(M)$ in the functional equation of the $L$-function of $M$ is given by the formula:

$$
\varepsilon(M)=\left|d_{k}\right|^{\frac{\operatorname{dim}(G)}{2}} f(M)^{\frac{1}{2}}
$$

where $d_{k}$ is the discriminant of $k$ over $\mathbb{Q}$. It also gives a proof of Theorem 11.5 when $k$ is a function field, assuming that $G$ has finite fundamental group of order prime to $\operatorname{char}(k)$, and putting $\left|d_{k}\right|=q^{2 g-2}$ as in [P].

\section{Mass Formulae}

We can use Proposition 9.3 to derive a number of explicit mass formulae. Let $k$ be a totally real number field, and let $G$ be a connected, reductive group over $k$, with $G(k \otimes \mathbb{R})=\prod_{v \mid \infty} G\left(k_{v}\right)$ compact. Recall that $M=\bigoplus_{d \geq 1} V_{d}(1-d)$, and let

$$
\Lambda(M, s)=\prod_{v} L_{v}(M, s)=\prod_{d \geq 1} \Lambda\left(V_{d}, s+1-d\right)
$$


be the global $L$-function of the motive $M$, so that

$$
\Lambda(M, s)=L_{\infty}(M, s) L(M, s)
$$

where $L(M, s)$ is the usual Artin $L$-function of $M$. We have Artin's functional equation [T, pg. 18-19]

$$
\Lambda(M, s)=\varepsilon(M, s) \Lambda\left(M^{\vee}, 1-s\right)
$$

with

$$
\varepsilon(M, s)=\left(\left|d_{k}\right|^{\operatorname{dim}(G)} f(M)\right)^{\frac{1}{2}-s} .
$$

In particular, taking $\Lambda(M)=\Lambda(M, 0)$, we find that

$$
\Lambda(M)=\left|d_{k}\right|^{\frac{\operatorname{dim}(G)}{2}} f(M)^{\frac{1}{2}} \Lambda\left(M^{\vee}(1)\right) .
$$

Now let $\mathbb{A}$ be the ring of adeles of $k$, and let $K=G(k \otimes \mathbb{R}) \times \prod_{v}$ finite $K_{v}$ be an open compact subgroup of $G(\mathbb{A})$. The double coset space

$$
\Sigma=G(k) \backslash G(\mathbb{A}) / K
$$

is then finite. If $\sigma \in \Sigma$, and $g \in G(\mathbb{A})$ represents the class of $\sigma$, then

$$
\Gamma_{\sigma}=G(k) \cap g K g^{-1}
$$

is a finite arithmetic subgroup of $G(k)$, of order $w_{\sigma}$. We define

$$
\operatorname{Mass}_{K}=\sum_{\sigma} \frac{1}{w_{\sigma}}
$$

where the sum is taken over all $\sigma$ in the double coset space $\Sigma$.

If $\mu_{K}$ is the unique Haar measure on the locally compact group $G(\mathbb{A})$ giving the open compact subgroup $K$ volume 1, then we also have

$$
\operatorname{Mass}_{K}=\int_{G(k) \backslash G(\mathbb{A})} \mu_{K} .
$$

Proposition 10.7. Assume that $G$ is quasi-split over $k_{v}$ for all finite places $v$, and that $K_{v}=\underline{G}^{0}\left(A_{v}\right) \subset G\left(k_{v}\right)$ is the special open compact subgroup defined in [Gr, $\S 4]$. Then,

$$
\operatorname{Mass}_{K}=\tau(G) \cdot \frac{1}{2^{n}} \cdot L(M)
$$

where $\tau(G)$ is the Tamagawa number of $G, n$ is the rank of the complex Lie group $G(k \otimes \mathbb{C})$, and $L(M)=L(M, 0)$.

Remarks. Note that if $l$ is the rank of $G$ over $k_{s}$ and $d$ is the degree of $k$ over $\mathbb{Q}$, then $n=l d$.

Proof. Let $\omega \neq 0$ be an invariant differential on $G$ over $k$, and $|\omega|_{v}$ the associated Haar measure on $G\left(k_{v}\right)$. For $v$ finite, if $G$ is unramified over $k_{v}$, with reductive model $\underline{G}$ over $A_{v}$, and $\omega$ has good reduction $\left(\bmod \pi_{v}\right)$, then,

$$
\int_{\underline{G}\left(A_{v}\right)} L_{v}\left(M^{\vee}(1)\right)|\omega|_{v}=1 .
$$

Hence the product

$$
\bigotimes_{v} L_{v}\left(M^{\vee}(1)\right)|\omega|_{v}
$$


defines a measure on $G(\mathbb{A})$. By definition, the Tamagawa measure $|\omega|_{\mathbb{A}}$ is given by

$$
|\omega|_{\mathbb{A}}=\frac{\bigotimes_{v} L_{v}\left(M^{\vee}(1)\right)|\omega|_{v}}{\left|d_{k}\right|^{\frac{\operatorname{dim}(G)}{2}} \Lambda\left(M^{\vee}(1)\right)} .
$$

Note that this is well-defined since the fact that $G(k \otimes \mathbb{R})$ is compact implies that $\Lambda\left(M^{\vee}(1)\right)$ is finite. Also, it is independent of the choice of $\omega \neq 0$. The Tamagawa number $\tau(G)$ is then defined by

$$
\tau(G)=\int_{G(k) \backslash G(\mathbb{A})}|\omega|_{\mathbb{A}} .
$$

On the other hand, the Haar measure $\mu_{K}$ on $G(\mathbb{A})$ is the product

$$
\mu_{K}=\mu_{G(k \otimes \mathbb{R})} \otimes \prod_{v \text { finite }}\left|\omega_{G_{v}}\right| L_{v}\left(M^{\vee}(1)\right)
$$

where $\mu_{G(k \otimes \mathbb{R})}$ is the measure giving $G(k \otimes \mathbb{R})$ volume 1 . Indeed, by Corollary 7.3, we have $\left|\omega_{G_{v}}\right|=\left|\omega_{G_{v}}^{\prime}\right|$, and the latter measure is constructed such that

$$
\int_{K_{v}}\left|\omega_{G_{v}}^{\prime}\right| L_{v}\left(M^{\vee}(1)\right)=1 .
$$

By $[\mathrm{Gr}, \S 7]$, we have

$$
\mu_{G(k \otimes \mathbb{R})} \cdot 2^{n} \prod_{v \mid \infty} L_{v}(M) e_{v}(G)=\prod_{v \mid \infty}\left|\omega_{G_{v}}\right| L_{v}\left(M^{\vee}(1)\right) .
$$

In fact, $\prod_{v \mid \infty} e_{v}(G)=1$ as $G$ is quasi-split at all finite places of $k$ (cf. [K]). Hence,

$$
\mu_{K}=2^{-n} \prod_{v \mid \infty} L_{v}(M)^{-1} \prod_{v}\left|\omega_{G_{v}}\right| L_{v}\left(M^{\vee}(1)\right) .
$$

By Proposition 9.3, we also have the formula

$$
\prod_{v} \frac{\left|\omega_{G_{v}}\right| L_{v}\left(M^{\vee}(1)\right)}{|\omega|_{v} L_{v}\left(M^{\vee}(1)\right)}=f(M)^{\frac{1}{2}}
$$

Hence, we have

$$
\begin{aligned}
\mu_{K} & =2^{-n} \prod_{v \mid \infty} L_{v}(M)^{-1} \cdot \Lambda\left(M^{\vee}(1)\right)\left|d_{k}\right|^{\frac{\operatorname{dim}(G)}{2}} f(M)^{\frac{1}{2}}|\omega|_{\mathbb{A}} \\
& =2^{-n} \prod_{v \mid \infty} L_{v}(M)^{-1} \cdot \Lambda(M)|\omega|_{\mathbb{A}} \\
& =2^{-n} L(M)|\omega|_{\mathbb{A}}
\end{aligned}
$$

and the mass formula follows from (10.9).

Even if $G$ is not quasi-split at all finite places $v$, one can obtain an explicit mass formula, by replacing $K_{v}$ at the bad primes by an Iwahori subgroup $J_{v} \subset G\left(k_{v}\right)$, and using Corollary 7.4. We leave the details to the reader. 


\section{REFERENCES}

[B-T] F. Bruhat and J. Tits, Groupes Reductifs sur un Corps Local I, IHES 41(1972), Pg 5-252; II, IHES 60(1984), Pg 5-184. MR 42:6245; MR 86c:20042

[Ch] C. Chevalley, Invariants of Finite Groups Generated by Reflections, Amer. J. Math. 77(1955), Pg 778-782. MR 17:345d

[Gr] B.H. Gross, On the Motive of a Reductive Group, Invent. Math. 130 (1997), 287-313. CMP 98:02

[K] R. Kottwitz, Sign Changes in Harmonic Analysis on Reductive Groups, Trans. AMS 278(1983), Pg 289-297. MR 84i:22012

[L] G. Laumon, Cohomology of Drinfeld Modular Varieties, Cambridge Studies in Advanced Math. 41(1996). MR 98c:11045a

[M-H] J. Milnor and D. Husemoller, Symmetric Bilinear Forms, Springer Ergebnisse 73(1973). MR 58:22129

[O] T. Ono, On the Arithmetic of Algebraic Tori, Annals of Math. 74(1961), Pg 101-139. MR 23:A1640

[P] G. Prasad, Volumes of S-arithmetic Quotients of Semisimple Groups, IHES 69(1989), Pg 91-117. MR 91c:22023

[Se] J.P. Serre, Linear Representations of Finite Groups, Springer GTM 42. MR 56:8675

[Se2] J.P. Serre, Conducteurs d'Artin des caracteres reels, Invent. Math. 14(1971), Pg 173-183. MR 48:273

[Se3] J.P. Serre, Local Fields, Springer GTM 67, 1979. MR 82e:12016

[Sp] T. Springer, Reductive Groups, in Proceedings of Symposium in Pure Math., Vol. 33, Part 1, Amer. Math. Soc., 1979. MR 80h:20062

[St] R. Steinberg, Endomorphisms of Linear Algebraic Groups, Memoirs AMS 80(1968). MR 37:6288

[T] J. Tate, Les conjectures des Stark sur les fonctions L d'Artin en s=0, Birkhäuser Progress in Math. 47(1984). MR 86e:12018a

Department of Mathematics, Harvard University, Cambridge, Massachusetts 02138

E-mail address: gross@math.harvard.edu

Department of Mathematics, Princeton University, Princeton, New Jersey 08540

E-mail address: wtgan@math.princeton.edu 